\title{
Development of Measurement and Analysis System for Electrolytic Cur- rent and Application to Multilayer Electrodeposition with a Coulomb Controller
}

\author{
Naoto TAKane, ${ }^{\mathrm{a}, \mathrm{b}, *}$ Hiroshi Narita, ${ }^{\mathrm{a}}$ and Susumu Arai ${ }^{\mathrm{b}}$ \\ ${ }^{a}$ Precision and Electronics Technology Department, Nagano Prefecture General Industrial Technology Center \\ (1-3-1, Osati-Katamacho, Okaya-shi, Nagano 394-0084, Japan) \\ ${ }^{\mathrm{b}}$ Faculty of Engineering, Shinshu University (4-17-1, Wakasato, Nagano-shi, Nagano 380-8553, Japan)
}

Received August 9, 2010 ; Accepted December 28, 2010

\begin{abstract}
In order to investigate the growth processes of nanometric multilayers electrodeposited using the single bath technique, we have developed a system that provides high-speed and high-resolution measurement and analysis of long time current. An attempt was made to analyze the current during $\mathrm{Co}-\mathrm{Cu} / \mathrm{Cu}$ multilayer electrodeposition using this system. Multilayers were electrodeposited using the single bath technique under potential control with a coulomb controller, and the target thickness of each layer $(5 \mathrm{~nm})$ was controlled according to the quantity of electricity. During electrodeposition of approximately $25 \mathrm{~min}$, the current was measured every $0.1 \mathrm{~ms}$ and analyzed. Cross-sectional observation by field emission-scanning electron microscopy (FE-SEM) showed a multilayered structure of which the layer thicknesses were close to $5 \mathrm{~nm}$. The quantities of electricity used for each layer were controlled exactly; however, the deposition times used varied for each Cu layer. The deposition time for each of the Co-Cu layers was constant; however, the current during each Co-Cu layer deposition was found to increase slowly. During electrodeposition, current peaks caused by overshooting were observed at every switching of the substrate potential. The estimated non-faradaic current included in the peak was sufficiently small with respect to a $5 \mathrm{~nm}$ layer thickness.
\end{abstract}

Key Words : Electrodeposition, Multilayers, Current Analysis, Coulomb Controller

\section{Introduction}

Electrodeposition has attracted much attention as a method for the fabrication of multilayered nanomaterials with electro-magneto ${ }^{1,2)}$ and mechanical ${ }^{3,4)}$ properties. The cost of electrodeposition is lower than vacuum technology and electrodeposition has some technological advantages, such as wide area deposition. The properties of multilayers are strongly dependent on the nanostructure; however, precise control of the structure in electrodeposited multilayers is difficult, because it is a complex deposition mechanism. Therefore, technological improvements in electrodeposition are required. The quality of electrodeposited multilayers is still inferior to analogs produced with vacuum technology. Although some studies ${ }^{5)}$ have characterized the structure of electrodeposited multilayers, problems with the electrodeposition technique are yet to be clarified.

The single bath technique is generally used for the electrodeposition of multilayers. This technique uses a single electrolyte solution containing two different metallic ions and a substrate potential at which only the nobler metal is electrodeposited, and one at which both of the metals are electrodeposited. Therefore, multilayers can be obtained by modulating the substrate potential during electrodeposition, so that layers electrodeposited at one of the potentials contain both of the metals. Generally, a step is taken that lessens the concentration of the nobler metal in the electrodeposited alloy by lessening the concentration of the nobler metallic ions in the solution. When the single bath technique is used, the current behavior is very complex, due to the wide difference between the concentrations of the two different metallic ions and the short periodic modulation of the substrate potential during long time electrodeposition. We have developed a coulomb controller ${ }^{6)}$ as a means to lessen the influence of current fluctuation that occurs during the single bath technique. This device lessens the fluctuation of layer thickness by controlling the quantity of electricity used for each nanometric layer during continuous potentiostatic electrodeposition with less than $1 \%$ error. Conventional devices, such as a coulometer, cannot perform such continuous and accurate control of the quantity of electricity carried by current with complex behavior. However, the current still fluctuates even with this device, and it is still important to obtain a detailed understanding of the growth process of electrodeposited multilayers in order to improve the quality of the structure.

Current analysis is an orthodox technique to investigate the processes involved in electrochemical reactions and also the growth processes of electrodeposited multilayers. ${ }^{7)}$ However, if current is measured at high-speed and high-resolution, the amount of measured data increases geometrically. Therefore, detailed analysis of the entire current during one multilayer electrodeposition has been difficult to obtain until now. As a solution to this problem, we have developed a system that provides high-speed and high-resolution measurement and analy- 
sis of long-time electrodeposition current in order to investigate nanometric multilayer growth processes during electrodeposition. Moreover, the current for multilayer electrodeposition by employing a coulomb controller was analyzed. An outline of the system is presented with results of the analysis.

\section{Experimental}

\section{1 Electrodeposition of multilayers}

$\mathrm{Co}-\mathrm{Cu} / \mathrm{Cu}$ multilayers, which is a popular multilayer system that has attracted attention as both an electromagneto ${ }^{1)}$ and mechanical ${ }^{4)}$ nanomaterial, were deposited in this investigation. Table 1 shows the plating bath conditions for electrodeposition. All chemicals used were reagent grade. The electrolyte solution was prepared with distilled water. Insoluble impurities were removed from the solution by filtration prior to the experiment. $\mathrm{Si}(100)$ wafers were used as substrates. A $50 \mathrm{~nm}$ thick Cr layer and then a $200 \mathrm{~nm}$ thick Au layer were evaporated onto the $\mathrm{Si}$ wafer. The substrate was cut and then masked with adhesive tape to produce an effective area of 0.655 $\mathrm{cm}^{2}$. The electrolytic cell used was a glass beaker with a $\mathrm{Pt}$ plate as the anode. The reference electrode was a saturated $\mathrm{Ag} / \mathrm{AgCl}$ electrode connected via a salt bridge. Prior to electrodeposition, the substrate was rinsed in pure water and diluted sulfuric acid. Table 2 shows the electrodeposition program used. Until the start of the electrodeposition program, the substrate potential was kept at the initial value $(0.00 \mathrm{~V}$ vs. $\mathrm{Ag} / \mathrm{AgCl})$ for a few seconds in the plating bath. After that, $20 \mathrm{Co}$-Cu layers and $20 \mathrm{Cu}$ layers, of which the target thicknesses were 5 $\mathrm{nm}$, were electrodeposited alternately. Moreover, three $\mathrm{Co}-\mathrm{Cu}$ layers and three $\mathrm{Cu}$ layers, of which the target thicknesses were almost $50 \mathrm{~nm}$, were electrodeposited alternately as a cover. The substrate potential was kept

Table 1 Plating bath conditions.

\begin{tabular}{cc}
\hline $\mathrm{Co}\left(\mathrm{SO}_{3} \mathrm{NH}_{2}\right)_{2} \cdot 4 \mathrm{H}_{2} \mathrm{O}$ & $0.62 \mathrm{~mol} \mathrm{dm}^{-3}$ \\
$\mathrm{CuSO}_{4} \cdot 5 \mathrm{H}_{2} \mathrm{O}$ & $0.0084 \mathrm{~mol} \mathrm{dm}^{-3}$ \\
Liquid Volume & $0.3 \mathrm{dm}^{3}$ \\
Temperature & $35-38^{\circ} \mathrm{C}$ \\
Agitation & None \\
$\mathrm{pH}$ & 1.7 (nonadjusted) \\
\hline
\end{tabular}

Table 2 Electrodeposition program.

\begin{tabular}{cccc}
\hline Step No. & $Q^{\mathrm{a})} / \mathrm{mC}$ & $E^{\mathrm{b})} / \mathrm{V}$ & Note \\
\hline- & - & 0.00 & Initial \\
\hline $1,3,5 \cdots 39$ & 9.537 & -0.95 & $\mathrm{Co}-\mathrm{Cu}, 5 \mathrm{~nm}$ \\
$2,4,6 \cdots 40$ & 8.914 & -0.55 & $\mathrm{Cu}, 5 \mathrm{~nm}$ \\
\hline $41,43,45$ & $92.25^{\mathrm{c}}$ & -0.95 & $\mathrm{Co}-\mathrm{Cu}$ \\
$42,44,46$ & $92.25^{\mathrm{c}}$ & -0.55 & $\mathrm{Cu}$ \\
\hline 47 & - & -0.75 & Final \\
\hline
\end{tabular}

${ }^{\text {a) }}$ Quantity of electricity. ${ }^{\text {b) }}$ Substrate potential vs. reference electrode $(\mathrm{Ag} / \mathrm{AgCl}) .{ }^{c}$ Steps Nos. $41-46$ are cover layers and 92.25 $\mathrm{mC}$ is equivalent to $\mathrm{Co}-48.36 \mathrm{~nm}$ or $\mathrm{Cu}-51.74 \mathrm{~nm}$. at the final value $(-0.75 \mathrm{~V}$ vs. $\mathrm{Ag} / \mathrm{AgCl})$ until the sample was removed from the plating bath after a few seconds from the start of step No. 47. The sample was then rinsed with pure water and dried. The substrate potentials of the $\mathrm{Co}-\mathrm{Cu}$ and $\mathrm{Co}$ layer electrodepositions were determined on the basis of the cathodic polarization curve. The thickness of each layer was controlled by the quantity of electricity used for electrodeposition; therefore, the set value for the quantity of electricity was calculated theoretically. This calculation assumed that all layers were pure metal with current efficiencies of 100 $\%$. The substrate potential was controlled using a potentiostat (Hokuto-Denko HA-501G), and the coulomb controller ${ }^{6}$ executed the electrodeposition program. The cathodic polarization curve was measured from the resting

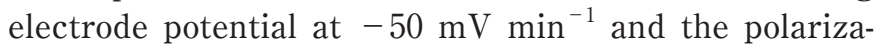
tion program was executed using a function generator (Hokuto-Denko HB-105).

The developed measurement system measured the current every $0.1 \mathrm{~ms}$ during electrodeposition. The measured data was processed by an analysis system. The current behavior in one layer was investigated, and the quantity of electricity and time required for each layer were determined. A cross-section of the multilayers was made using a cross-section polisher (Jeol SM-09010) and the cross-section was observed by field emission-scanning microscopy (FE-SEM; Hitachi S-5200). During crosssection sample preparation, the sample was heated several times to approximately $120-130^{\circ} \mathrm{C}$ to fix on several jigs using hot wax. In addition, the preliminary crosssection, which was to be finished by the cross-section polisher, was prepared by machining operations of cutting and lapping, which is a standard process for using a cross-section polisher. Delamination of the electrodeposited film was not observed during experiments, even during preparation of the cross-section samples.

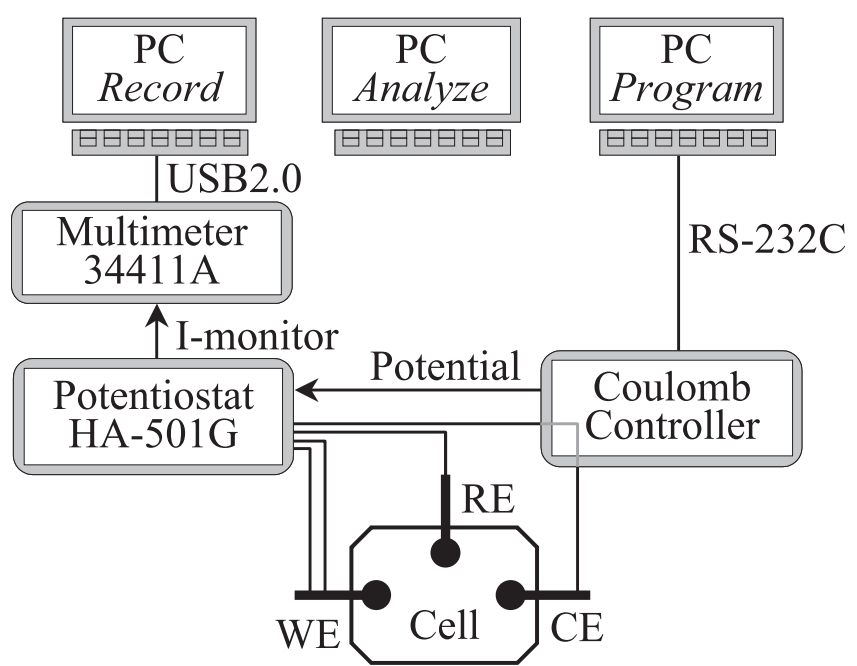

Fig. 1 Schematic illustration of the experimental equipment setup. The coulomb controller executes the electrodeposition program with monitoring of the current passing through the plating bath. The multimeter and the connected PC constitute the measurement system that measures and records the current in detail. 


\section{2 Measurement system}

The experimental equipment employed is shown in Fig. 1. A 6-1/2 digital multimeter (Agilent 34411A) was used to measure the current. This multimeter has high accuracy for not only the measured value, but also the sampling rate; therefore, it is suitable for analysis of the quantity of electricity. The multimeter was used to measure the output voltage of the current monitor terminal of the potentiostat.

The measured data was temporarily stored in the internal memory of the multimeter. A personal computer (PC) periodically acquired the stored data. The multimeter is an independent device with a sufficiently fast interface; therefore, the experimental method could involve continual exact measurement, and the processing performance of the $\mathrm{PC}$ does not influence the measurement. The minimum sampling interval of this system is $20 \mu \mathrm{s}$ and measurements can be made for a few hours even at the minimum sampling interval.

\section{3 Analysis system}

The analysis software was developed in-house and was used to process the measured data. The entire measured data (original data) includes a large number of data points, so that analysis by graphing with all data points is almost impossible. Therefore, the analysis software processes the original data to extract the data points that express the outline of the original data. The extracted points (outline data) can be used to determine characteristic points of the original data. Generally, expected variations of electrochemical current are A) significant variations caused by transient phenomena, and B) slow variations caused by fluctuations of the steady

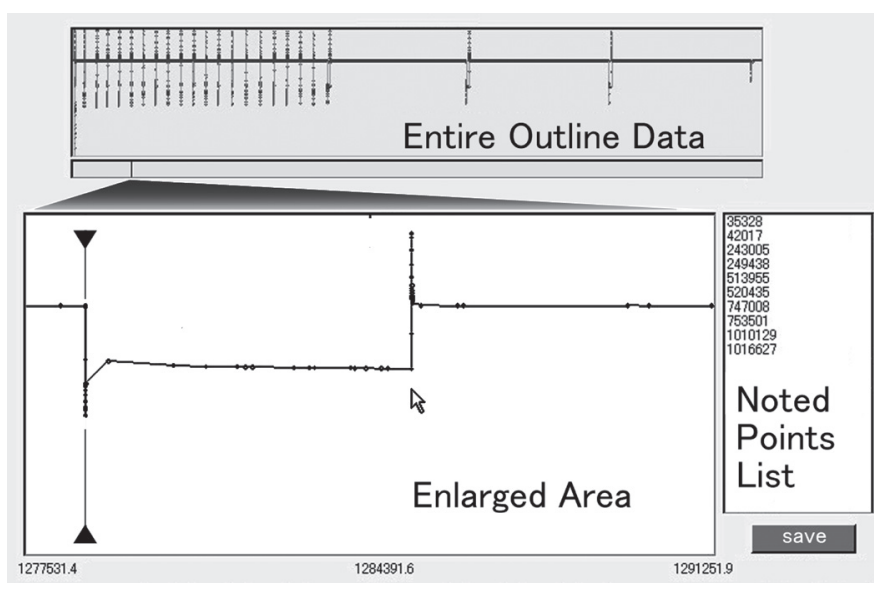

Fig. 2 Window of the analysis software. This software produces an outline graph of the measured current, as shown in the figure. The user can select and note the characteristic points of the measured current containing a large number of data points by clicking in the enlarged area. Detailed analysis can then be performed using the noted points. The graph indicated in this figure is the outline data of this experiment. The vertical axis unit is arbitrary, but proportional to the current, and the upper side is positive. The horizontal axis indicates the serial number of data points of the original data and is proportional to time. state. In the case of multilayer electrodeposition, an example of A) is a current variation caused by switching of the substrate potential, and an example of B) is a current variation caused by fluctuation of the solution convection.

In order to detect A), namely significant variations, the analysis software extracts the data points at which the curvatures of the graph are large. At every data point of the original data, the analysis software calculates the average of the previous point and the next point, and the difference between the data point and this average. The software extracts the points at which the absolute value of this difference is larger than a certain threshold. For this experiment, the target number of extracted points in this process was set to 10000 . The analysis software calculates the threshold automatically before this process so that the number of extracted points is close to the target number.

In order to detect B) variations, the analysis software performs re-sampling of the original data as data thinning. In this process, a random sampling method is used in order to avoid noise known as folding noise, which is caused by interference between the signal and sampling frequencies. The target number of extracted points in this process was also set to 10000 .

The analysis software generates one outline data by putting together the points extracted as A) and B). As shown in Fig. 2, it produces a graph of outline data and the user can select any data points on the graph (noted point). The user can extract any part of the original data including a noted point by using the noted point as an index. With this function, any specific part of the original data can be analyzed in detail. Moreover, the analysis software calculates the quantity of electricity between a pair of noted points by integrating the original data. By selecting moments of transitions of the steps as noted points, the quantity of electricity and the time used for each step can be determined.

\section{Results}

A cathodic polarization curve is shown in Fig. 3. The reduction of $\mathrm{Cu}$ ions begins at around $0.00 \mathrm{~V}$ vs. $\mathrm{Ag} /$ $\mathrm{AgCl}$ and then reaches a limiting current density. On the other hand, the reduction of $\mathrm{Co}$ ions begins at around $-0.70 \mathrm{~V}$ vs. $\mathrm{Ag} / \mathrm{AgCl}$ and then the current increases monotonically. Therefore, Fig. 3 shows that the rate-determining step at the potential of $\mathrm{Cu}$ layers shown in Table 2 is the diffusion of $\mathrm{Cu}$ ions, and the rate-determining step of the $\mathrm{Co}-\mathrm{Cu}$ layers is the activation reaction. Figure 4 shows a cross-section of the deposited multilayers. Some voids are present between the substrate and multilayers, but a multilayered structure that is close to the design was achieved.

Figure 5 shows a part of the outline data generated by the analysis system and the corresponding part of the original data. The original data was $1.5 \times 10^{7}$ points in this experiment. The amount of outline data is almost $0.16 \%$ of the original data, but it can express both outline and details of the original data. The quantities of electricity for each layer were calculated by the analysis 


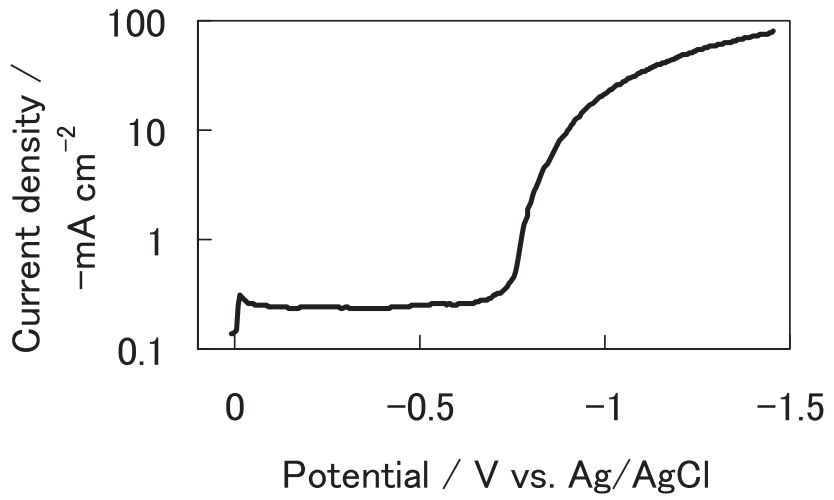

Fig. 3 Cathodic polarization curve of $0.62 \mathrm{~mol} \mathrm{dm}^{-3}$ $\mathrm{Co}\left(\mathrm{SO}_{3} \mathrm{NH}_{2}\right)_{2} \cdot 4 \mathrm{H}_{2} \mathrm{O}+0.0084 \mathrm{~mol} \mathrm{dm}{ }^{-3} \mathrm{CuSO}_{4} \cdot 5 \mathrm{H}_{2} \mathrm{O}$ measured without agitation. The scan rate was $-50 \mathrm{mV} \mathrm{\textrm {min } ^ { - 1 }}$ and the working electrode was a $\mathrm{Si}$ wafer on which $\mathrm{Cr}$ and then $\mathrm{Au}$ were evaporated. The conditions for the electrolyte solution and electrodes were with the same as those for electrodeposition of the multilayer sample.

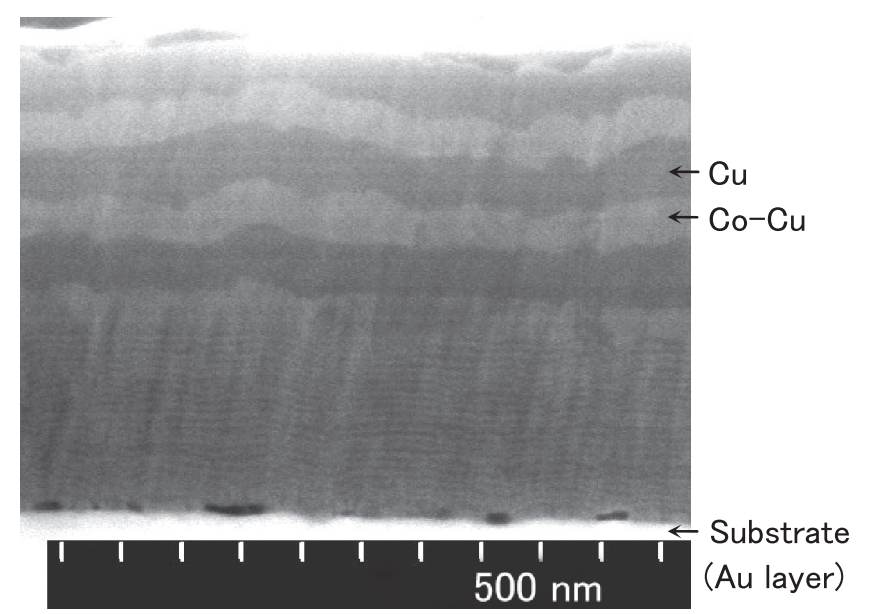

Fig. 4 Cross-sectional FE-SEM image of the multilayer electrodeposited sample. The sample was prepared using a cross-section polisher.

system, and the errors were less than $0.5 \%$ against the set values shown in Table 2. These errors are lower than the allowable error of the coulomb controller. ${ }^{6)}$ The analysis system also calculated the deposition time for each $5 \mathrm{~nm}$ layer of Nos. 1-40, and the results are shown in Fig. 6. The deposition time of the $\mathrm{Co}-\mathrm{Cu}$ layers was constant, but that of the $\mathrm{Cu}$ layers was not, which indicates that the mean current for the $\mathrm{Co}-\mathrm{Cu}$ layer was constant but that for the $\mathrm{Cu}$ layer was not, because the quantity of electricity was fixed.

As shown in Fig. 5, the current peaks caused by overshoot were observed at switching of the substrate potential. Similar peaks occurred at every switching of the substrate potential during electrodeposition. Figure 7 shows an enlarged peak at the start of step No. 3 during $\mathrm{Co}-\mathrm{Cu}$ layer electrodeposition. Figure 8 shows that for step No. 4 during $\mathrm{Cu}$ layer electrodeposition. The forms of the peaks at the start of $\mathrm{Cu}$ layer deposition were relatively simple, but those for $\mathrm{Co}-\mathrm{Cu}$ layer deposition were

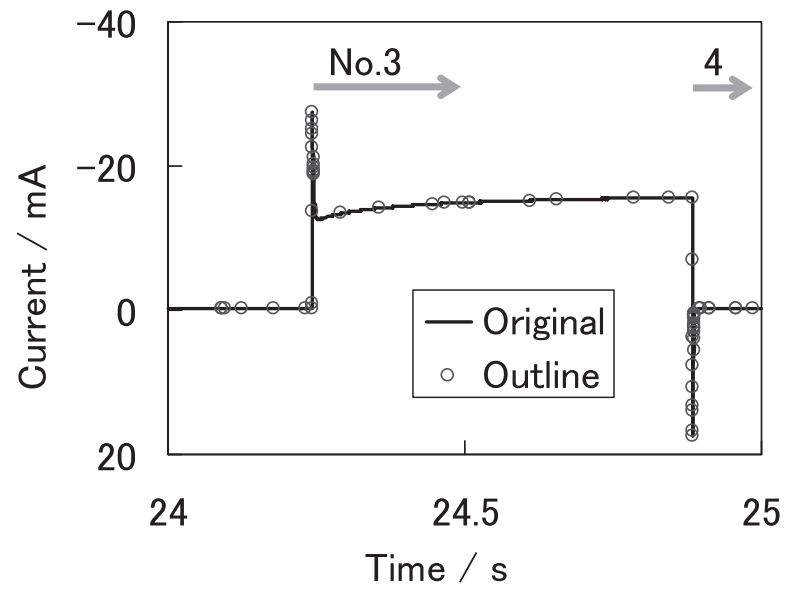

Fig. 5 Part of the original data and outline data of the current around step No. 3 during electrodeposition of the multilayer sample. The original data is raw data recorded by the measurement system and the outline data is generated from the original data by the analysis system.

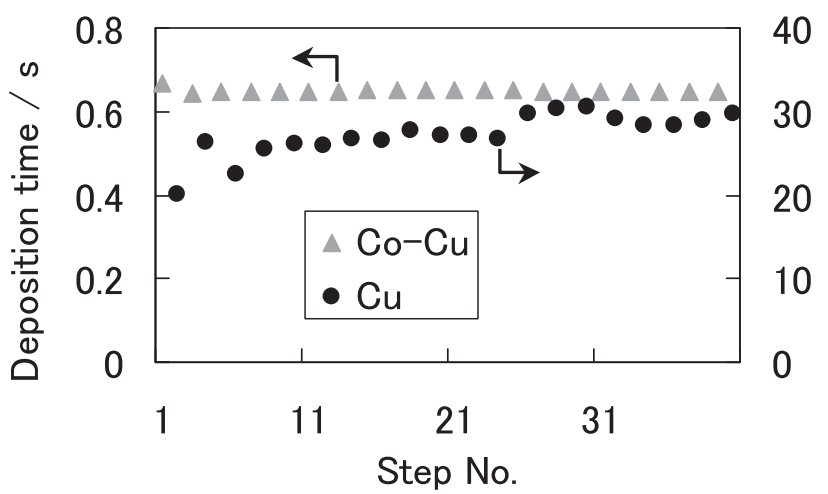

Fig. 6 Deposition time for each $5 \mathrm{~nm}$ layer of Nos. 1-40 in the multilayer sample. Using the analysis system, the transition moments of the electrodeposition step were selected from the measured current data as the noted points. The deposition times shown are the intervals of the noted points.

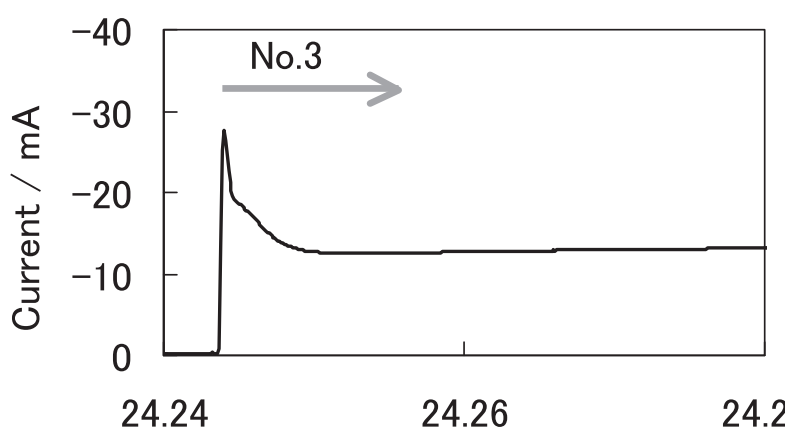

Time / s

Fig. 7 Current transition at the beginning of step No. 3, which is typical of a $5 \mathrm{~nm}$ Co-Cu electrodeposition layer in the multilayer sample. The substrate potential was changed from -0.55 to $-0.95 \mathrm{~V}$ vs. $\mathrm{Ag} / \mathrm{AgCl}$ at the start of step No. 3 . 


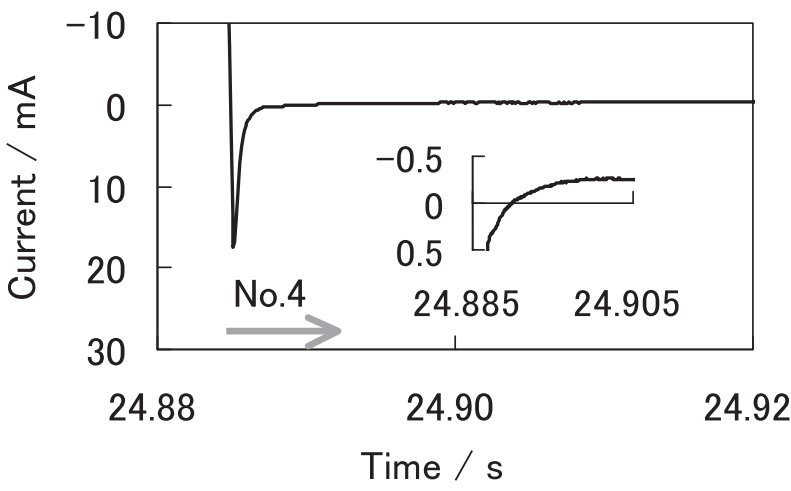

Fig. 8 Current transition at the beginning of step No. 4 , which is typical of a $5 \mathrm{~nm} \mathrm{Cu}$ electrodeposition layer in the multilayer sample. The inset shows an enlarged part of the graph. The substrate potential was changed from -0.95 to $-0.55 \mathrm{~V}$ vs. $\mathrm{Ag} / \mathrm{AgCl}$ at the start of step No. 4 .

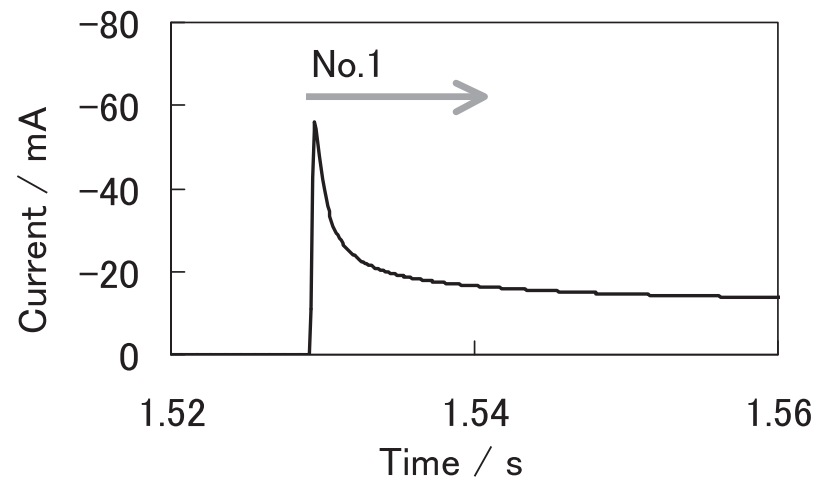

Fig. 9 Current transition at the beginning of step No. 1, which is the first $5 \mathrm{~nm} \mathrm{Co-Cu}$ electrodeposition layer in the multilayer sample. The substrate potential was changed from 0.00 to $-0.95 \mathrm{~V}$ vs. $\mathrm{Ag} / \mathrm{AgCl}$ at the start of step No. 1.

complex, as shown in Fig. 7 and 8. In Fig. 8, the peak area is $13.6 \mu \mathrm{C}$ when the constant current at $24.904 \mathrm{~s}$ is considered as the baseline. The peak areas for the $5 \mathrm{~nm}$ $\mathrm{Cu}$ layers among Nos. 2-40 were determined in the same way to be $P_{\mathrm{Cu}}=13.1 \pm 0.8 \mu \mathrm{C}$ and constant. The peak areas for Nos. 42,44 and 46 of the cover $\mathrm{Cu}$ layers were 13.8, 14.2 and $13.9 \mu \mathrm{C}$, respectively, which were almost the same as that for $P_{\mathrm{Cu}}$ of the $5 \mathrm{~nm} \mathrm{Cu}$ layers. The peak areas of the $\mathrm{Co}-\mathrm{Cu}$ layers were not determined, due to the complex waveform, but the peak forms and sizes of all the Co-Cu layers except step No. 1 were almost the same as that in Fig. 7. The peak of step No. 1 is shown in Fig. 9 and was larger than that shown in Fig. 7. The peak of the final step (No. 47) is shown in Fig. 10. Step No. 47 is the transition from the potential of $\mathrm{Cu}$ layer electrodeposition to around the equilibrium potential of Co. The peak area in Fig. 10 was $5.43 \mu \mathrm{C}$ when the constant current at $1496.517 \mathrm{~s}$ was considered as the baseline.

The deposition time of the Co-Cu layer deposition was relatively constant, as shown in Fig. 6. However, the current during each $\mathrm{Co}-\mathrm{Cu}$ layer deposition varied, as

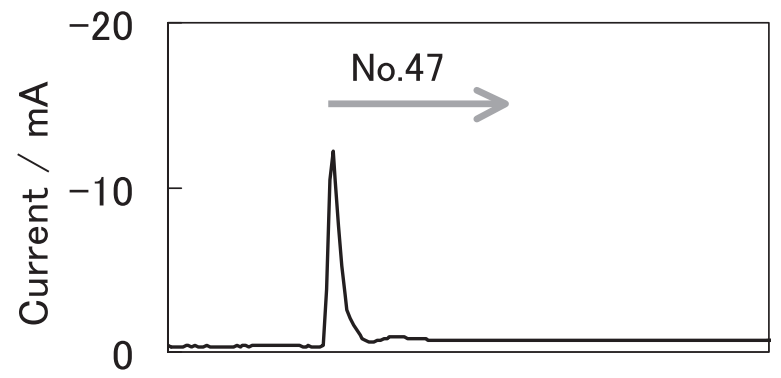

1496.51

1496.53

Time / s

Fig. 10 Current transition at the beginning of step No. 47, which is the final electrodeposition step of the multilayer sample. The substrate potential was changed from -0.55 to $-0.75 \mathrm{~V}$ vs. $\mathrm{Ag} / \mathrm{AgCl}$ at the start of step No. 47 .

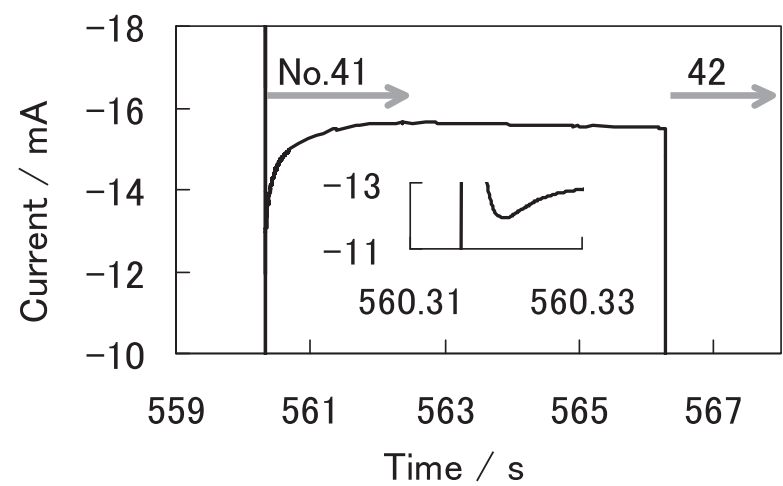

Fig. 11 Current transition during step No. 41, which is the electrodeposition of the first cover layer of almost 50 $\mathrm{nm} \mathrm{Co-Cu}$ in the multilayer sample. The inset shows an enlarged part of the graph. The substrate potential was changed from -0.55 to $-0.95 \mathrm{~V}$ vs. $\mathrm{Ag} / \mathrm{AgCl}$ at the start of step No. 41.

shown in Fig. 5. The current during the $\mathrm{Co}-\mathrm{Cu}$ layer deposition increased slowly after the peak, and was still increasing at the end of step No. 3. The same behavior was observed in every $\mathrm{Co}-\mathrm{Cu}$ layer. Figure 11 shows the current for step No. 41, which is a thicker Co-Cu layer. The current increased by $30 \%$ from the minimum value and reached an almost constant value after more than 1 s from the start of step No. 41. On the other hand, the average current for each $\mathrm{Cu}$ layer varied, as shown in Fig. 6. The current for each $\mathrm{Cu}$ layer varied randomly; no systematic tendency of current variation or similarity between each $\mathrm{Cu}$ step was observed.

\section{Discussion}

Without preliminary experiments for control of the layer thickness, a multilayered structure close to that of the design was obtained, which indicates that the current efficiencies were relatively high in this experiment. When the single bath technique is used, layer thickness is usually controlled by deposition time. Therefore, deposition rates are determined by preliminary experiments and deposition times are calculated from the deposition 
rates and target values of layer thickness. However, as shown in Fig. 5 and 6, because the electrodeposition currents for both the $\mathrm{Co}-\mathrm{Cu}$ and $\mathrm{Cu}$ layers were not constant, the estimated deposition rates were also variable. Therefore, the coulomb control method, which can avoid the influence of current fluctuation, is appropriate for multilayer electrodeposition, rather than the preliminary determination of the deposition rates. The repeatability of the deposition rate among $\mathrm{Cu}$ layers was estimated to be considerably low from Fig. 6. The rate-determining step of $\mathrm{Cu}$ layer electrodeposition is the diffusion of $\mathrm{Cu}$ ions; therefore, the electrodeposition rate would be strongly influenced by variation of the $\mathrm{Cu}$ ion diffusion layer and the fluctuation of convection in the solution.

The current peaks that occurred at switching of the substrate potential in this experiment contain both transitions of faradaic and non-faradaic current. Generally, the former is caused by a transition of the diffusion layer of ions on the electrode, and the latter is caused by charge or discharge of the electric double layer. In the coulomb control method, non-faradaic current is a direct cause of error. Therefore, it is important to estimate the capacitance of the electric double layer. Step No. 47 was investigated to estimate the capacitance of the electric double layer, although the current peak of Step No. 47 may contain both faradaic and non-faradaic current. However, Fig. 3 implies that the steady faradaic current at $-0.75 \mathrm{~V}$ vs. $\mathrm{Ag} / \mathrm{AgCl}$, which is the substrate potential of step No. 47, was sufficiently small for the concentration of $\mathrm{Co}$ ions in the solution. Moreover, the reduction of $\mathrm{Cu}$ ions already reached the limiting current density. Therefore, the diffusion layer of ions on the substrate at step No. 47 should be almost the same as that at step No. 46. Consequently, the faradaic current transition at the beginning of step No. 47 should cause only an insignificant peak. This means that almost all of the peak area shown in Fig. 10 can be regarded as non-faradaic current. By dividing the peak area by the width of the substrate potential variation $(0.20 \mathrm{~V})$, the capacitance of the electric double layer is estimated to be approximately $C_{\mathrm{d}}$ $=27 \mu \mathrm{F}$. If the capacitance of the electric double layer is $C_{\mathrm{d}}$ and fixed, then the non-faradaic current during each layer electrodeposition should be $P_{\mathrm{es}}=11 \mu \mathrm{C}$ in this experiment, because the width of the substrate potential variation was $0.40 \mathrm{~V}$. The peak area of $P_{\mathrm{Cu}}=13.1$ $\pm 0.8 \mu \mathrm{C}$ observed at $\mathrm{Cu}$ layers is close to $P_{\mathrm{es}}=11 \mu \mathrm{C}$, which implies that the capacitance of the electric double layer was, even for $\mathrm{Cu}$ layer electrodeposition, close to $C_{\mathrm{d}}=27 \mu \mathrm{F} . P_{\mathrm{Cu}}=13.1 \pm 0.8 \mu \mathrm{C}$ and $P_{\mathrm{es}}=11 \mu \mathrm{C}$ are only approximately $0.1 \%$ of the quantities of electricity for the $5 \mathrm{~nm}$ layers shown in Table 2 and are sufficiently small in this experiment. The form of the peak shown in Fig. 7 implies that this peak includes an effect that is similar to the reverse of the peak shown in Fig. 8. Therefore, even for the Co-Cu layers, except step No. 1, the quantity of non-faradaic current was probably close to $P_{\mathrm{es}}=11 \mu \mathrm{C}$. Even the entire area of the peak shown in Fig. 7 is only a few times as large as $P_{\mathrm{Cu}}$ or $P_{\mathrm{es}}$ and this is also sufficiently small in this experiment.

As shown in Fig. 4, the undulation of the cover layers is large. The geometric area of the cathode seems to be increased with growth of the film. However, the current peak areas showed no significant variation among the $\mathrm{Cu}$ layers including step Nos. 42, 44 and 46. The magnitude of the sample surface undulation shown in Fig. 4 may be insufficient to increase the practical area of the electric double layer.

The peak area of $P_{\mathrm{Cu}}=13.1 \pm 0.8 \mu \mathrm{C}$ observed for $\mathrm{Cu}$ layers is slightly larger than $P_{\mathrm{es}}=11 \mu \mathrm{C}$ estimated in this section. The causes for this difference between $P_{\mathrm{Cu}}$ and $P_{\mathrm{es}}$ may be the variation in the electric double layer capacity and/or mere experimental errors. However, the oxidation current may be one of the causes. At the beginning of $\mathrm{Cu}$ layer electrodeposition, the surface of the substrate was a Co-Cu layer, and the substrate potential was nobler than the potential at which Co deposition began in Fig. 3. Therefore, $\mathrm{Co}$ in the $\mathrm{Co}-\mathrm{Cu}$ layer may have been oxidized. However, this oxidation current would have to be sufficiently small, because $P_{\mathrm{Cu}}$ was small and the difference between $P_{\mathrm{Cu}}$ and $P_{\mathrm{es}}$ was also small. Co oxidation itself can also be caused by displacement of $\mathrm{Cu}$ ions. This phenomenon can also cause errors in a multilayered structure; however, investigation of this phenomenon was difficult in this experiment. This phenomenon will need to be investigated in the future using other techniques.

The peak of step No. 1 shown in Fig. 9 was larger than that of the other steps. One of the causes of this is the width of the substrate potential variation, which was larger than that of the other steps. This peak probably also includes the variation of faradaic current caused by the formation of the diffusion layer of $\mathrm{Cu}$ ions. If the voids shown in Fig. 4 were on the surface at the beginning of electrodeposition, then they would influence the current by the variation of the electric double layer capacitance. However, to estimate the influence of the voids in this experiment was difficult, due to other influences such as faradaic current variation.

The form of the Co-Cu layer electrodeposition peak, except step No. 1, is complex, as shown in Fig. 7. The cause of this complexity can be regarded as a decrease of faradaic current. Although the rate-determining step is the activation reaction, the relatively large current can decrease slightly, due to enlargement of the diffusion layer. Figure 7 implies that this decrease of current was finished within several milliseconds, after which, the current increased for a relatively long time. The rate-determining step of the $\mathrm{Co}-\mathrm{Cu}$ layer (activation reaction) implies that this current increase is caused by a variation of overpotential. Figure 3 shows that the current increase shown in Fig. 11 corresponds to decreases in overpotential by $30-50 \mathrm{mV}$. The decrease in overpotential can be modeled based on the change of the cathode surface. At the beginning of $\mathrm{Co}-\mathrm{Cu}$ electrodeposition, the cathode surface was $\mathrm{Cu}$. Co electrodeposition on $\mathrm{Cu}$ increases the overpotential, due to the difference between the substrate and the electrodeposited material. The overpotential should then decrease with formation of the $\mathrm{Co}-\mathrm{Cu}$ layer. According to this model, Fig. 5 and $11 \mathrm{im}-$ ply that the $5 \mathrm{~nm}$ Co-Cu layer was discontinuous in this 
experiment and that the under $\mathrm{Cu}$ layer was exposed. $\mathrm{Co}-\mathrm{Cu}$ layer continuity could not be verified in this experiment. In the future, this continuity will have to be investigated using other analytical techniques, such as cross-sectional observation using transmission electron microscopy (TEM). According to this model, the overpotential should also vary during $\mathrm{Cu}$ layer electrodeposition. However, the rate-determining step of $\mathrm{Cu}$ layer electrodeposition was the diffusion of $\mathrm{Cu}$ ions and the current was almost constant around the potential of $\mathrm{Cu}$ layer electrodeposition, as shown in Fig. 3. This implies that the correlation between the current and the potential was extremely weak around that potential, although the current and the potential correlate in principle. The weak correlation was probably the reason why the current behavior caused by the overpotential variation was not observed during $\mathrm{Cu}$ layer electrodeposition in this experiment.

\section{Conclusion}

We have developed a system that provides high-speed and high-resolution measurement and analysis of the current during nanometric multilayer electrodeposition. The current behavior during multilayer electrodeposition was investigated and analyzed over a long time period, in addition to sudden current behavior within highresolution intervals. The following conclusions were made from this analysis.

1) When $\mathrm{Co}-\mathrm{Cu} / \mathrm{Cu}$ multilayers were electrodeposited by the coulomb control method, the deposition time for the $\mathrm{Co}-\mathrm{Cu}$ layer was constant, but that for the $\mathrm{Cu}$ layer was not.

2) The current during each $\mathrm{Co}-\mathrm{Cu}$ layer deposition was not constant and increased slowly.

3) Current peaks caused by overshoot were observed at every switching of the substrate potential.

4) The estimated non-faradaic current included in the peak at switching of the substrate potential was sufficiently small for $5 \mathrm{~nm}$ layer electrodeposition in this experiment.

This system allows the precise analysis of current for practical multilayer electrodeposition. An example analysis using this system found several noteworthy phenomena that might influence multilayered structures. We intend to improve and develop the technology of multilayer electrodeposition using this system.

\section{Acknowledgments}

The authors acknowledge Mika Makimura and Yutaka Komatsu of the Nagano prefecture Industrial Technology Center for their help in the cross-sectional FE-SEM observations.

\section{References}

1) Á. Cziráki, M. Köteles, L. Péter, Z. Kupay, J. Pádár, L. Pogány, I. Bakonyi, M. Uhlemann, M. Herrich, B. Arnold, J. Thomas, H. D. Bauer, and K. Wetzig, Thin Solid Films, 433, 237 (2003).

2) S. Kainuma, S. Ishikura, and K. Hisatake, J. Magn. Soc. Jpn., 21, 557 (1997) [in Japanese].

3) T. Miyake, M. Kume, K. Yamaguchi, D. P. Amalnerkar, and H. Minoura, Thin Solid Films, 397, 83 (2001).

4) T. Hattori, Y. Kaneko, and S. Hashimoto, J. Jpn. Inst. Met., 73, 306 (2009) [in Japanese].

5) A. Bartók, A. Csik, K. Vad, G. Molnár, E. Tóth-Kádár, and L. Péter, J. Electrochem. Soc., 156, D253 (2009).

6) N. Takane and H. Narita, Electrochemistry, 75, 879 (2007) [in Japanese].

7) N. V. Myung and K. Nobe, Plat. Surf. Finish., 87, 125 (2000). 